\title{
Duality in a special class of submanifolds and Frobenius manifolds
}

\author{
O.I. Mokhov
}

Consider totally nonisotropic $N$-dimensional submanifolds $M^{N}$ in $(N+L)$-dimensional pseudo-Euclidean spaces $\mathbb{E}_{k}^{N+L}(N$-dimensional submanifolds that are not tangent to isotropic cones of the ambient $(N+L)$-dimensional pseudo-Euclidean space at their points).

Let $\left(z^{1}, \ldots, z^{N+L}\right)$ be pseudo-Euclidean coordinates in $\mathbb{E}_{k}^{N+L}$; a submanifold $M^{N}$ is given by a smooth vector-function $r=\left(z^{1}\left(u^{1}, \ldots, u^{N}\right), \ldots, z^{N+L}\left(u^{1}, \ldots, u^{N}\right)\right)$, $\operatorname{rank}\left(\partial z^{i} / \partial u^{j}\right)=N, 1 \leq i \leq N+L, 1 \leq j \leq N ; \partial r / \partial u^{i}=r_{i}(u), 1 \leq i \leq N$, is a basis of the tangent space at an arbitrary point $u=\left(u^{1}, \ldots, u^{N}\right)$ of the submanifold $M^{N} ; n_{1}(u), \ldots, n_{L}(u)$ is an arbitrary basis of the normal space $\mathbb{N}_{u}$ that depends smoothly on the point $u ; g_{i j}(u)=\left(r_{i}, r_{j}\right), 1 \leq i, j \leq N$, is the first fundamental form of the submanifold $((\cdot, \cdot)$ is the pseudo-Euclidean scalar product in $\left.\mathbb{E}_{k}^{N+L}\right), h_{\alpha \beta}(u)=\left(n_{\alpha}, n_{\beta}\right), 1 \leq \alpha, \beta \leq L\left(\operatorname{det} g_{i j}(u) \neq 0\right.$ and $\operatorname{det} h_{\alpha \beta}(u) \neq 0$ for totally nonisotropic submanifolds). The Gauss and Weingarten decompositions have the form

$$
\frac{\partial^{2} r}{\partial u^{i} \partial u^{j}}=a_{i j}^{k}(u) \frac{\partial r}{\partial u^{k}}+b_{i j}^{\beta}(u) n_{\beta}(u), \quad \frac{\partial n_{\alpha}}{\partial u^{j}}=c_{\alpha j}^{k}(u) \frac{\partial r}{\partial u^{k}}+d_{\alpha j}^{\beta}(u) n_{\beta}(u),
$$

respectively. The coefficients $a_{i j}^{k}(u), b_{i j}^{\beta}(u), c_{\alpha j}^{k}(u), d_{\alpha j}^{\beta}(u)$, the metric $g_{i j}(u)$ and the functions $h_{\alpha \beta}(u)$ satisfy a number of relations including the Gauss equations, the Codazzi equations and the Ricci equations for any submanifold. Note that in this paper we consider only the local theory of submanifolds.

We shall single out a special class of $N$-dimensional submanifolds in $2 N$-dimensional pseudo-Euclidean spaces. Our purpose is to single out the case, when the sets of the basis vectors of the tangent and normal spaces, $r_{i}(u), 1 \leq i \leq N$, and $n_{\alpha}(u)$, $1 \leq \alpha \leq L$, possess equal rights and are dual one to another. It is obvious that the condition $L=N$ is necessary for such duality. Moreover, another necessary condition is the potentiality of the basis $n_{\alpha}(u): n_{\alpha}(u)=\partial n / \partial u^{\alpha}, 1 \leq \alpha \leq N$, where $n(u)$ is a certain vector-function on the submanifold.

Definition 1. A basis $n_{\alpha}(u), 1 \leq \alpha \leq N$, in the normal space $\mathbb{N}_{u}$ of a certain $N$-dimensional submanifold in a $2 N$-dimensional pseudo-Euclidean space is called

\footnotetext{
${ }^{1}$ The work was completed with the financial support of the Russian Foundation for Basic Research (grant no. 08-01-00054) and the Programme for Support of Leading Scientific Schools (grant no. NSh-1824.2008.1).
} 
potential if there exists a vector-function (a potential of the normals) $n(u)$ on the submanifold such that $n_{\alpha}(u)=\partial n / \partial u^{\alpha}, 1 \leq \alpha \leq N$.

If the vector-function $n(u)$ exists, then it generates a potential basis in the normal space $\mathbb{N}_{u}$ in any coordinate system.

Definition 2. An $N$-dimensional submanifold in a $2 N$-dimensional pseudoEuclidean space is called a submanifold with potential normal basis (or a submanifold with potential of normals) if there exists a vector-function (a potential of normals) $n(u)$ on the submanifold such that the vectors $n_{i}(u)=\partial n / \partial u^{i}, 1 \leq i \leq N$, form a basis in the normal space $\mathbb{N}_{u}$ at any point $u$ on the submanifold.

Definition 2 is invariant (it does not depend on a local coordinate system).

The coefficients $a_{i j}^{k}(u)$ in the Gauss decomposition (1) are always the coefficients of the Levi-Civita connection of the metric $g_{i j}(u)$. If the submanifold is equipped with a potential normal basis, then a similar assertion is also true for the coefficients $d_{\alpha j}^{\beta}(u)$ in the Weingarten decomposition (1). Consider the corresponding Gauss and Weingarten decompositions:

$$
\frac{\partial^{2} r}{\partial u^{i} \partial u^{j}}=a_{i j}^{k}(u) \frac{\partial r}{\partial u^{k}}+b_{i j}^{k}(u) \frac{\partial n}{\partial u^{k}}, \quad \frac{\partial^{2} n}{\partial u^{i} \partial u^{j}}=c_{i j}^{k}(u) \frac{\partial r}{\partial u^{k}}+d_{i j}^{k}(u) \frac{\partial n}{\partial u^{k}} .
$$

Theorem 1. The functions $h_{i j}(u)=\left(\partial n / \partial u^{i}, \partial n / \partial u^{j}\right)$ define a covariant metric on the submanifold, and the coefficients $d_{i j}^{k}(u)$ in the Weingarten decomposition (2) are the coefficients of the symmetric affine connection compatible with the metric $h_{i j}(u)$, that is the coefficients of the Levi-Civita connection of the metric $h_{i j}(u)$. The coefficients $b_{i j}^{k}(u)$ and $c_{i j}^{k}(u)$ are tensors of type $(1,2)$ symmetric with respect to the subscripts $i$ and $j$ on the submanifold $M^{N}$.

Theorem 2 (duality principle). If a submanifold is equipped with a potential of normals $n(u)$ and given by the vector-functions $(r(u), n(u))$, then the vector-functions $(n(u), r(u))$ also give a submanifold equipped with the potential of normals $r(u)$ such that $\partial n / \partial u^{i}, 1 \leq i \leq N$, are tangent vectors and $\partial r / \partial u^{i}, 1 \leq i \leq N$, are basis normal vectors of the submanifold, and moreover, in this case all the objects of the local theory of such submanifolds are dual to each other, in particular, the Gauss decomposition becomes the Weingarten decomposition and the Weingarten decomposition becomes the Gauss decomposition, the Gauss equations becomes the Ricci equations and the Ricci equations becomes the Gauss equations, the Codazzi equations changes to themselves (they are self-dual), the tensor $b_{i j}^{k}(u)$ becomes the tensor $c_{i j}^{k}(u)$ and the tensor $c_{i j}^{k}(u)$ becomes the tensor $b_{i j}^{k}(u)$, the metric $g_{i j}(u)$ becomes the metric $h_{i j}(u)$ and vice versa.

Submanifolds with potential of normals form an important and rich class of submanifolds. Arbitrary one-dimensional submanifolds of pseudo-Euclidean planes are a trivial example of such submanifolds. The general theory of submanifolds with potential of normals, the duality principle for them and important examples will be presented in a separate paper. The submanifolds equipped with natural Frobenius 
structures, which were constructed by the present author in [1]-[5] and which realize arbitrary Frobenius manifolds (the theory of Frobenius manifolds was constructed in [6]), are a particular case of submanifolds with potential of normals. The present author has proved in [1] and [2] that an arbitrary Frobenius manifold can be realized as a certain flat submanifold with potential of normals for which $g_{i j}(u)=\operatorname{ch}_{i j}(u)$, $c=$ const $\neq 0$, where $c$ is a deformation parameter preserving the corresponding Frobenius structure. Let $h_{i j}(u)=\eta_{i j}, \eta_{i j}=\eta_{j i}$, det $\eta_{i j} \neq 0, \eta_{i j}=$ const, $g_{i j}=c \eta_{i j}$, $c=$ const $\neq 0$. In this case, for the submanifolds with potential of normals, the relations $a_{i j}^{k}(u)=d_{i j}^{k}(u)=0$ are satisfied, and also there exists a function $\Phi(u)$ such that $b_{i j}^{k}(u)=\eta^{k s} \partial^{3} \Phi / \partial u^{s} \partial u^{i} \partial u^{j}, c_{i j}^{k}(u)=-(1 / c) b_{i j}^{k}(u), \eta^{i s} \eta_{s j}=\delta_{j}^{i}$, and all the relations of the local theory of submanifolds are satisfied if and only if the function $\Phi(u)$ satisfies the associativity equations of two-dimensional topological quantum field theories (the Witten-Dijkgraaf-Verlinde-Verlinde equations, see [6])

$$
\frac{\partial^{3} \Phi}{\partial u^{i} \partial u^{j} \partial u^{s}} \eta^{s p} \frac{\partial^{3} \Phi}{\partial u^{p} \partial u^{k} \partial u^{l}}=\frac{\partial^{3} \Phi}{\partial u^{i} \partial u^{k} \partial u^{s}} \eta^{s p} \frac{\partial^{3} \Phi}{\partial u^{p} \partial u^{j} \partial u^{l}}
$$

\section{Bibliography}

[1] O.I. Mokhov, Frobenius manifolds as a special class of submanifolds in pseudoEuclidean spaces, will be published in AMS Translations Series 2, Volume 224, Advances in Mathematical Sciences, eds. V.M.Buchstaber and I.M.Krichever, "Geometry, Topology, and Mathematical Physics: S.P.Novikov's Seminar: 2006-2007", Amer. Math. Soc., Providence, RI 2008; http://arXiv.org/abs/0710.5860 (2007).

[2] O.I. Mokhov, Theory of submanifolds, the associativity equations in 2D topological quantum field theories, and Frobenius manifolds, In: Proceedings of the Workshop "Nonlinear Physics. Theory and Experiment. IV." (Gallipoli, Lecce, Italy, 22 June - 1 July, 2006), published in Teoret. i Matemat. Fizika 152:2 (2007), 368-376; English transl., Theoret. Math. Phys. 152:2 (2007), 1183-1190; Preprint MPI 06-152, Max-Planck-Institut für Mathematik, Bonn, Germany, 2006; arXiv:math.DG/0610933 (2006).

[3] O.I. Mokhov, Submanifolds in pseudo-Euclidean spaces and Dubovin-Frobenius structures, In: Proceedings of the 10th International Confrence "Differential Geometry and its Applications" in honour of the 300th anniversary of the birth of Leonhard Euler, August 27-31, 2007, Olomouc, Czech Republic, World Scientific, Singapore, 2008, pp. 505-516.

[4] O.I. Mokhov, Non-local Hamiltonian operators of hydrodynamic type with flat metrics, and the associativity equations, Uspekhi Mat. Nauk 59:1 (2004), 187-188; English transl., Russian Math. Surveys 59:1 (2004), 191-192. 
[5] O.I. Mokhov, Nonlocal Hamiltonian operators of hydrodynamic type with flat metrics, integrable hierarchies, and the associativity equations, Funktsional. Anal. i Prilozhen. 40:1 (2006), 14-29; English transl., Funct. Anal. Appl. 40:1 (2006), 11-23; arXiv:hep-th/0406292 (2004).

[6] B. Dubrovin, Geometry of 2D topological field theories, In: Lecture Notes in Math., vol. 1620, Springer-Verlag, Berlin, 1996, pp. 120-348; arXiv:hep-th/9407018 (1994).

\section{O.I. Mokhov}

Centre for Non-Linear Studies,

Landau Institute for Theoretical Physics,

Russian Academy of Sciences;

Department of Geometry and Topology, Faculty of Mechanics and Mathematics,

Lomonosov Moscow State University

E-mail: mokhov@mi.ras.ru; mokhov@landau.ac.ru; mokhov@bk.ru 